\title{
Studies on Sequence Distributions of Terpolyesters by High-Resolution Nuclear Magnetic Resonance
}

\author{
Masao Murano \\ Katata Research Institute, Toyobo Company Ltd., \\ Katata, Otsu, Japan.
}

(Received June 6, 1970)

\begin{abstract}
NMR spectra of poly(ethylene terephthalate--sebacate-fumarate) and poly(ethylene terephthalate-sebacate-malonate) terpolyesters, which are obtained by ordinary molten-state polycondensation under reduced pressure, are studied. Ethylene glycol protons show six kinds of dyad sequences and their areas are measured by a curve resolver. Their relative intensities are in good accord with the calculated values for random (or Bernoullian) terpolymers. The relationships of three kinds of combination of two acid groups are plotted in the triangular coordinate system by normalizing the three kinds of dyad sequences. It is found that these three acid groups are randomly distributed.
\end{abstract}

KEY WORDS Sequence Distribution / NMR / Terpolymer / Polyester / Triangular Coordinate System / Curve Resolver / Poly(Ethylene Terephthalate-Sebacate-Fumarate) / Poly(Ethylene TerephthalateSebacate-Malonate)/

High-resolution nuclear magnetic resonance (NMR) is becoming an increasingly important tool in the study of sequence distributions in copolymers since its first application was demonstrated by Ferguson, ${ }^{1}$ and many vinyl copolymers have been studied. But as for condensation copolymers, there are only a few reports about their sequence distributions by NMR. ${ }^{2-5}$

The authors ${ }^{21}$ studied the sequence distributions of many kinds of copolyesters prepared by ordinary molten-state polycondensation from ethylene glycol and dimethyl esters of dicarboxylic acids, and found many of them were random (or Bernoullian) copolymers from their ethylene glycol proton resonance. The transesterification reaction between two kinds of homopolyesters was also studied and it was found that random (or Bernoullian) copolyesters were formed by this reaction. ${ }^{2,6}$

As found in our binary copolyester, glycol units are sensitive to the linking acid groups and show three kinds of linkages in glycol units, $\mathrm{A}-\mathrm{E}-\mathrm{A}, \mathrm{A}-\mathrm{E}-\mathrm{B}$ (or $\mathrm{B}-\mathrm{E}-\mathrm{A}$ ), and $\mathrm{B}-\mathrm{E}-\mathrm{B}$, where $A$ and $B$ represent acid groups and $E$ glycol units.
In terpolyesters prepared from ethylene glycol and three kinds of acids, six kinds of linkages, $\mathrm{A}-\mathrm{E}-\mathrm{A}, \mathrm{A}-\mathrm{E}-\mathrm{B}$ (or $\mathrm{B}-\mathrm{E}-\mathrm{A}$ ), $\mathrm{A}-\mathrm{E}-\mathrm{C}$ (or $\mathrm{C}-\mathrm{E}-\mathrm{A}$ ), $\mathrm{B}-\mathrm{E}-\mathrm{B}, \mathrm{B}-\mathrm{E}-\mathrm{C}$ (or $\mathrm{C}-\mathrm{E}-\mathrm{B}$ ), and $\mathrm{C}-\mathrm{E}-\mathrm{C}$, are found in their ethylene glycol proton resonance. ${ }^{7}$

In the present paper, the sequence distributions of many kinds of terpolyesters, prepared by ordinary molten-state polycondensation from ethylene glycol and dimethylesters of dicarboxylic acids, are studied by NMR spectra.

\section{EXPERIMENTAL}

Poly(ethylene terephthalate-sebacate-fumarate), $\mathrm{PET} / \mathrm{S} / \mathrm{F}$, and poly(ethylene terephthalate - sebacate-malonate), PET/S/M, were prepared from ethylene glycol and dimethyl esters of the corresponding acids by ordinary molten-state polycondensation under reduced pressure at $195^{\circ} \mathrm{C}$. Binary copolyesters and homopolyesters were also prepared from the corresponding dimethyl esters.

NMR spectra were measured in chloroform solution $(0.05 \mathrm{~g} / \mathrm{m} l)$ at $70^{\circ} \mathrm{C}$ with Varian A-60 and HA-100 spectrometers and the spectra were resolved by a du Pont 310 Curve Resolver. 


\section{RESULTS AND DISCUSSION}

When we make a terpolymer from $\mathrm{A}, \mathrm{B}$, and $C$ comonomers, it has 9 kinds of dyad sequence, $\mathrm{A}-\mathrm{A}, \mathrm{A}-\mathrm{B}, \mathrm{A}-\mathrm{C}, \mathrm{B}-\mathrm{A}, \mathrm{B}-\mathrm{B}, \mathrm{B}-\mathrm{C}, \mathrm{C}-\mathrm{A}$, $\mathbf{C}-\mathbf{B}$, and $\mathbf{C}-\mathrm{C}$, and in the case of terpolyesters consisting of three kinds of acids and ethylene glycol, they have six dyad sequences, $\mathrm{A}-\mathrm{E}-\mathrm{A}$, $\mathrm{A}-\mathrm{E}-\mathrm{B}$ ( or $\mathrm{B}-\mathrm{E}-\mathrm{A}), \mathrm{A}-\mathrm{E}-\mathrm{C}$ (or $\mathrm{C}-\mathrm{E}-\mathrm{A}$ ), $\mathrm{B}-\mathrm{E}-\mathrm{B}, \mathrm{B}-\mathrm{E}-\mathrm{C}$ (or $\mathrm{C}-\mathrm{E}-\mathrm{B}$ ), and $\mathrm{C}-\mathrm{E}-\mathrm{C}$, where $\mathrm{A}, \mathrm{B}, \mathrm{C}$ represent acid groups and $\mathrm{E}$ ethylene glycol units.

In Figure 1, 60-MHz NMR spectra of PET/ S/F terpolyesters are shown and ethylene glycol protons have six resonance peaks. The binary copolymers, PET $/ \mathrm{S}, \mathrm{PES} / \mathrm{F}$, and $\mathrm{PEF} / \mathrm{T}$, show three resonance peaks in the ethylene glycol region, and the homopolymers, PET, PES, and PEF have one peak, respectively. Therefore, these six peaks in PET/S/F terpolyesters can be assigned to $T-E-T, T-E-F$ (or $F-E-T$ ), $T-E-S$ (or $S-E-T$ ), F-E-F, S-E-F (or F-E-S), and $S-E-S$ linkages from the low field, considering the NMR spectra of binary copolymers and homopolymers. These assignments are summarized in Table I.

NMR spectra of PET/S/M terpolyesters were also shown in Figure 2 and six resonance peaks in ethylene glycol protons were assigned in Table II.

These six peaks in ethylene glycol protons of $\mathrm{PET} / \mathrm{S} / \mathrm{F}$ were resolved with a du Pont 310 Curve Resolver, overlapping six Lorentzian curves as shown in Figure 3. PET/S/M terpolyesters are

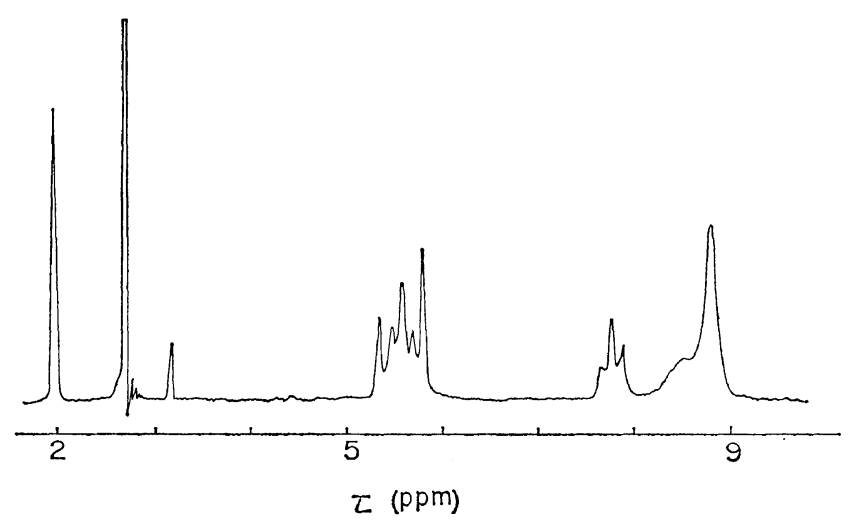

(a)

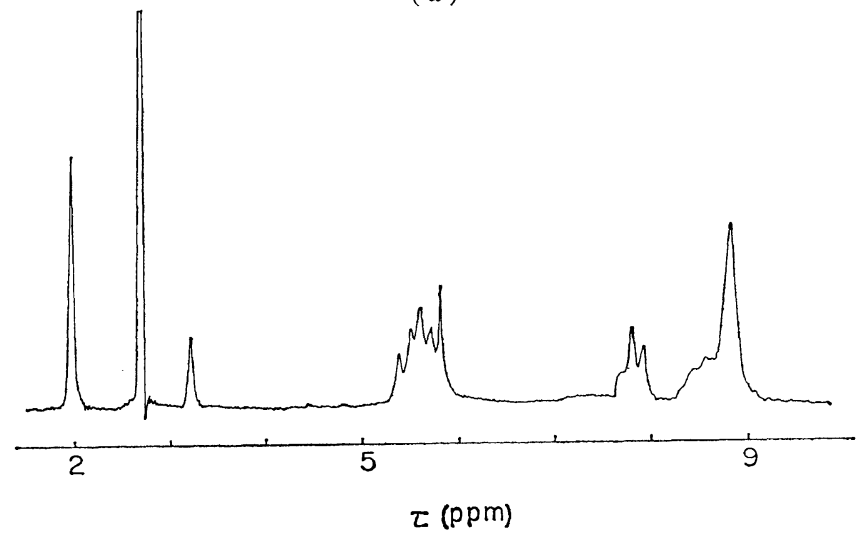

(b)

Figure 1. 60-MHz NMR spectra of PET/S/F terpolymers ((a) 40/40/20 and (b) $35 / 35 / 30$ ) in chloroform at $70^{\circ} \mathrm{C}$. 
Table I. Assignments of ethylene glycol proton signals in $\mathrm{PET} / \mathrm{S} / \mathrm{F}$ terpolymers

\begin{tabular}{ll}
\hline T-E-T & $5.38 \mathrm{ppm}$ \\
$\mathrm{T}-\mathrm{E}-\mathrm{F}$ & 5.50 \\
$\mathrm{~T}-\mathrm{E}-\mathrm{S}$ & 5.60 \\
$\mathrm{~F}-\mathrm{E}-\mathrm{F}$ & 5.61 \\
$\mathrm{~S}-\mathrm{E}-\mathrm{F}$ & 5.72 \\
$\mathrm{~S}-\mathrm{E}-\mathrm{S}$ & 5.82 \\
\hline
\end{tabular}

Table II. Assignments of ethylene glycol proton signals in $\mathrm{PET} / \mathrm{S} / \mathrm{M}$ terpolymers

\begin{tabular}{ll}
\hline T-E-T & $5.38 \mathrm{ppm}$ \\
T-E-M & 5.55 \\
T-E-S & 5.60 \\
M-E-M & 5.72 \\
M-E-S & 5.77 \\
S-E-S & 5.82 \\
\hline
\end{tabular}

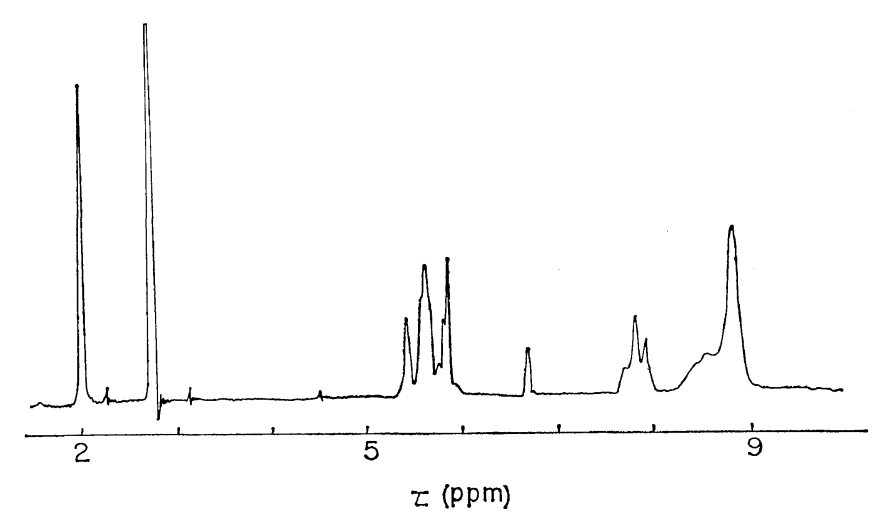

(a)

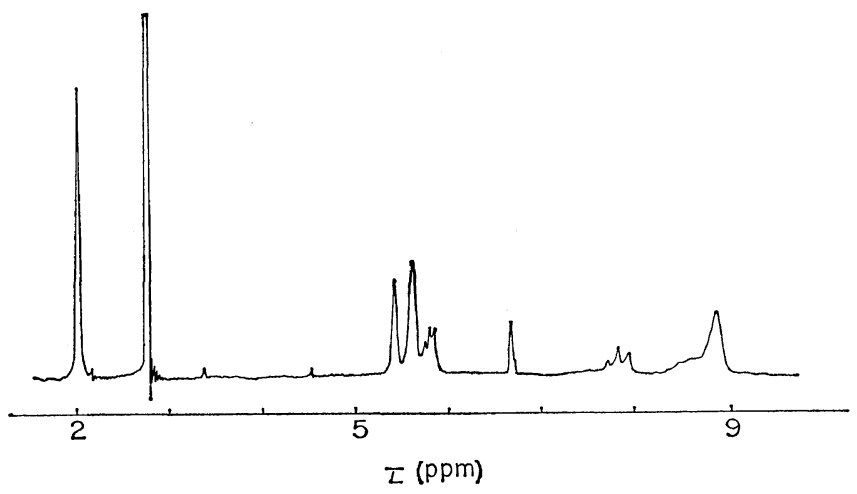

(b)

Figure 2. 60-MHz NMR spectra of PET/S/M terpolymers ((a) 40/40/20 and (b) $50 / 20 / 30$ ) in chloroform at $70^{\circ} \mathrm{C}$.

also resolved in Figure 4, and the area of each peak was measured by the integrator.

From these values of six peaks, the degree of randomness of the terpolymers can be discussed. Molar fractions of terephthalate $\left(\boldsymbol{P}_{\mathrm{T}}\right)$, sebacate $\left(\boldsymbol{P}_{\mathrm{S}}\right)$, and fumarate $\left(\boldsymbol{P}_{\mathrm{F}}\right)$ are obtained from the intensities of the six kinds of signals in ethylene glycol protons by eq 1, 2, and 3, as well as from their relative intensities of aro- matic protons of terephthalate, methylene protons of sebacate, and methine protons of fumarate.

$$
\begin{aligned}
& \boldsymbol{P}_{\mathrm{T}}=\boldsymbol{P}_{\mathrm{T}-\mathrm{E}-\mathrm{T}}+1 / 2 \boldsymbol{P}_{\mathrm{T}-\mathrm{E}-\mathrm{S}}+1 / 2 \boldsymbol{P}_{\mathrm{T}-\mathrm{E}-\mathrm{F}} \\
& \boldsymbol{P}_{\mathrm{S}}=\boldsymbol{P}_{\mathrm{S}-\mathrm{E}-\mathrm{S}}+1 / 2 \boldsymbol{P}_{\mathrm{T}-\mathrm{E}-\mathrm{S}}+1 / 2 \boldsymbol{P}_{\mathrm{S}-\mathrm{E}-\mathrm{F}} \\
& \boldsymbol{P}_{\mathrm{F}}=\boldsymbol{P}_{\mathrm{F}-\mathrm{E}-\mathrm{F}}+1 / 2 \boldsymbol{P}_{\mathrm{T}-\mathrm{E}-\mathrm{F}}+1 / 2 \boldsymbol{P}_{\mathrm{S}-\mathrm{E}-\mathrm{F}}
\end{aligned}
$$

where $\quad \boldsymbol{P}_{\mathrm{T}-\mathrm{E}-\mathrm{T}}, \quad \boldsymbol{P}_{\mathrm{T}-\mathrm{E}-\mathrm{S}}, \quad \boldsymbol{P}_{\mathrm{T}-\mathrm{E}-\mathrm{F}}, \quad \boldsymbol{P}_{\mathrm{S}-\mathrm{E}-\mathrm{S}}$, $\boldsymbol{P}_{\mathrm{S}-\mathrm{E}-\mathrm{F}}$, and $\boldsymbol{P}_{\mathrm{F}-\mathrm{E}-\mathrm{F}}$ represent the proportions of the integrated intensities of the corresponding 
Sequence Distributions of Terpolyesters by NMR

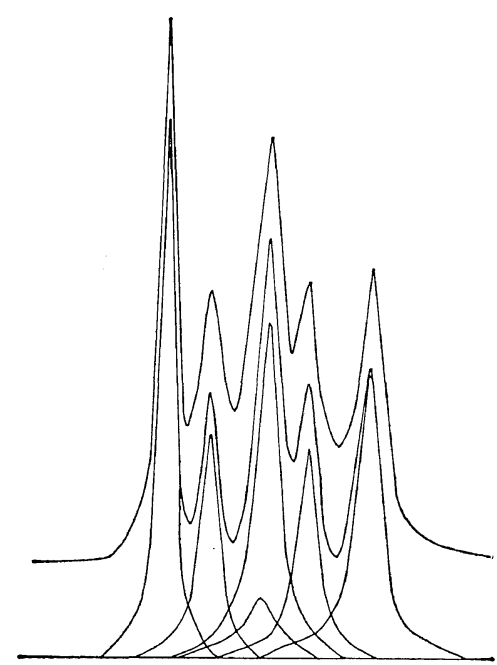

(a)

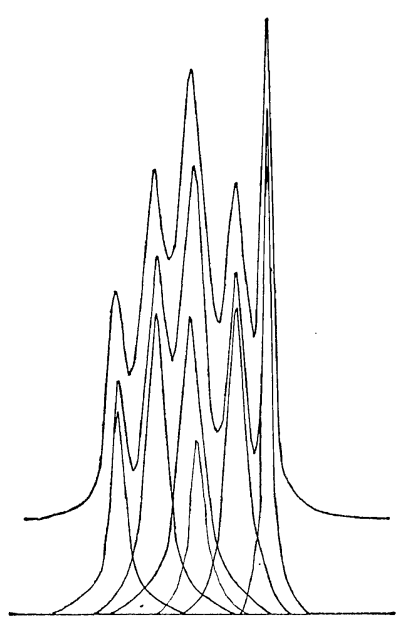

(b)

Figure 3. Ethylene glycol proton resonance spectra of PET/S/F terpolymers ((a) 40/40/20 and (b) 35/ $35 / 30)$; upper, observed spectra; middle, synthesized spectra; lower, six Lorentzian curves.

peaks of ethylene glycol protons.

The probability of finding a $\mathrm{T}$ unit next to an $\mathrm{S}$ unit in $\mathrm{PET} / \mathrm{S} / \mathrm{F}$ terpolyester can be obtained by eq 4 ,

$$
\begin{aligned}
\boldsymbol{P}_{\mathrm{ST}} & =\frac{1 / 2 \boldsymbol{P}_{\mathrm{S}-\mathrm{E}-\mathrm{T}}}{\boldsymbol{P}_{\mathrm{S}-\mathrm{E}-\mathrm{S}}+1 / 2 \boldsymbol{P}_{\mathrm{S}-\mathrm{E}-\mathrm{T}}+1 / 2 \boldsymbol{P}_{\mathrm{S}-\mathrm{E}-\mathrm{T}}} \\
& =\frac{\boldsymbol{P}_{\mathrm{S}-\mathrm{E}-\mathrm{T}}}{2 \boldsymbol{P}_{\mathrm{S}}}
\end{aligned}
$$

Similarly, $\boldsymbol{P}_{\mathrm{TS}}, \boldsymbol{P}_{\mathrm{TF}}, \boldsymbol{P}_{\mathrm{FT}}, \boldsymbol{P}_{\mathrm{SF}}$, and $\boldsymbol{P}_{\mathrm{FS}}$ can be

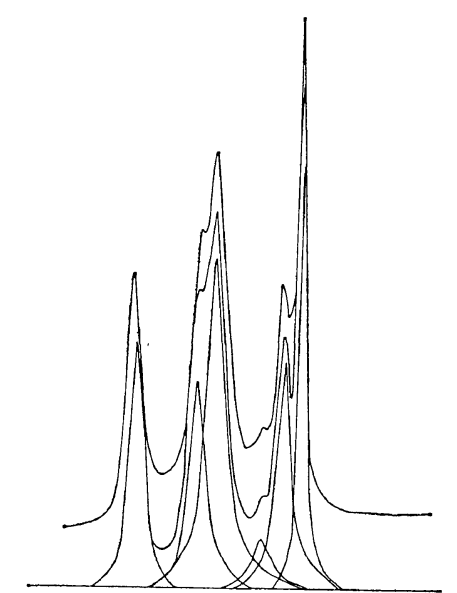

(a)

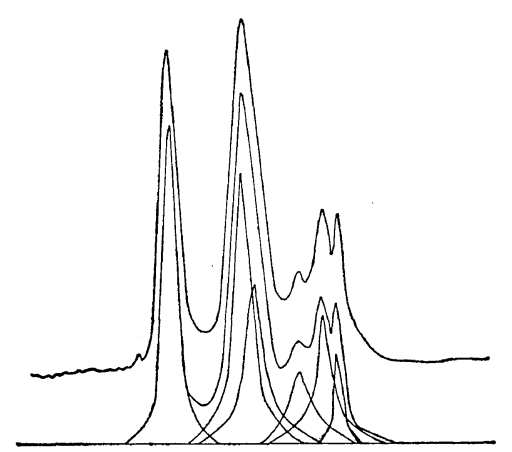

(b)

Figure 4. Ethylene glycol proton resonance spectra of PET/S/M terpolymers ((a) 40/40/20 and (b) 50/ 20/30); upper, observed spectra; middle, synthesized spectra; lower, six Lorentzian curves.

obtained by eq $5-9$,

$$
\begin{aligned}
& \boldsymbol{P}_{\mathrm{TS}}=\frac{\boldsymbol{P}_{\mathrm{T}-\mathrm{E}-\mathrm{S}}}{2 \boldsymbol{P}_{\mathrm{T}}} \\
& \boldsymbol{P}_{\mathrm{TF}}=\frac{\boldsymbol{P}_{\mathrm{T}-\mathrm{E}-\mathrm{F}}}{2 \boldsymbol{P}_{\mathrm{T}}} \\
& \boldsymbol{P}_{\mathrm{FT}}=\frac{\boldsymbol{P}_{\mathrm{F}-\mathrm{E}-\mathrm{T}}}{2 \boldsymbol{P}_{\mathrm{F}}} \\
& \boldsymbol{P}_{\mathrm{SF}}=\frac{\boldsymbol{P}_{\mathrm{S}-\mathrm{E}-\mathrm{F}}}{2 \boldsymbol{P}_{\mathrm{S}}} \\
& \boldsymbol{P}_{\mathrm{FS}}=\frac{\boldsymbol{P}_{\mathrm{F}-\mathrm{E}-\mathrm{S}}}{2 \boldsymbol{P}_{\mathrm{F}}}
\end{aligned}
$$

And the degree of randomness is defined by eq 10 , 


\section{Murano}

$$
\boldsymbol{B}=\boldsymbol{P}_{\mathrm{ST}}+\boldsymbol{P}_{\mathrm{TS}}+\boldsymbol{P}_{\mathrm{TF}}+\boldsymbol{P}_{\mathrm{FT}}+\boldsymbol{P}_{\mathrm{SF}}+\boldsymbol{P}_{\mathrm{FS}}
$$

In the case of a random (or Bernoullian) terpolymer, where $\boldsymbol{P}_{\mathrm{ST}}=\boldsymbol{P}_{\mathrm{FT}}=\boldsymbol{P}_{\mathrm{T}}, \boldsymbol{P}_{\mathrm{TS}}=\boldsymbol{P}_{\mathrm{FS}}=$ $\boldsymbol{P}_{\mathrm{S}}$, and $\boldsymbol{P}_{\mathrm{TF}}=\boldsymbol{P}_{\mathrm{SF}}=\boldsymbol{P}_{\mathrm{F}}, \boldsymbol{B}$ can be written in eq 11 ,

$$
\boldsymbol{B}=2\left(\boldsymbol{P}_{\mathrm{T}}+\boldsymbol{P}_{\mathrm{S}}+\boldsymbol{P}_{\mathrm{F}}\right)=2
$$

So a random terpolymer takes 2 as the value of $B$.

These values are shown in Table III. The same calculations were applied to PET/S/M terpolyesters and are shown in Table IV. The calculated values of six dyad distributions, assuming random (or Bernoullian) distribution of three acid groups, are also shown in the Tables and the measured values are in good accord with the calculated values. $B$ takes almost 2, which means these acid units are randomly distributed in these terpolyesters.

The degree of randomness can also be evaluated by other methods. ${ }^{8-10}$ Chujo ${ }^{8}$ proposed the quantity $\Delta \varepsilon$ to classify the polymerization mechanism, and in the same way the degree of randomness is obtained by eq. 12, for PET/S. copolymer,

$$
R=\ln \frac{\boldsymbol{P}_{\mathrm{TT}} \cdot \boldsymbol{P}_{\mathrm{SS}}}{\boldsymbol{P}_{\mathrm{TS}} \cdot \boldsymbol{P}_{\mathrm{ST}}}=\ln \frac{\left(1-\boldsymbol{P}_{\mathrm{TS}}\right)\left(1-\boldsymbol{P}_{\mathrm{ST}}\right)}{\boldsymbol{P}_{\mathrm{TS}} \cdot \boldsymbol{P}_{\mathrm{ST}}}
$$

In the random (or Bernoullian) copolymer, where $\boldsymbol{P}_{\mathrm{TS}}=\boldsymbol{P}_{\mathrm{S}}$, and $\boldsymbol{P}_{\mathrm{ST}}=\boldsymbol{P}_{\mathrm{T}}$, or $\boldsymbol{P}_{\mathrm{T}-\mathrm{E}-\mathrm{S}}=2 \boldsymbol{P}_{\mathrm{T}} \cdot \boldsymbol{P}_{\mathrm{S}}$, $R$ takes 0 . In an alternative copolymer, $R$ takes $-\infty$ and it becomes larger according to the increase in block parts of the copolymers. For $\mathrm{PET} / \mathrm{S} / \mathrm{F}$ terpolymer $R$ can be obtained by $\mathrm{eq}$ 13 ,

\begin{tabular}{|c|c|c|c|c|c|c|c|c|}
\hline Sample & $\begin{array}{l}\text { Feed ratios, } \\
\mathrm{T} / \mathrm{S} / \mathrm{F}\end{array}$ & $\mathrm{T}-\mathrm{E}-\mathrm{T}$, & $\mathrm{T}-\underset{\%}{\mathrm{E}-\mathrm{F}}$ & $\underset{\%}{\mathrm{~T}-\mathrm{E}-\mathrm{S},}$ & $\mathrm{F}-\underset{\%}{\mathrm{E}-\mathrm{F}}$ & $\mathrm{S}-\underset{\%}{\mathrm{E}-\mathrm{F}}$, & $\mathbf{S}-\underset{\%}{\mathrm{E}-\mathrm{S}} \mathrm{S}$, & $B$ \\
\hline 1 & $45 / 45 / 10$ & $\begin{array}{r}20.45 \\
(20.25)\end{array}$ & $\begin{array}{r}9.09 \\
(9.00)\end{array}$ & $\begin{array}{r}40.90 \\
(40.50)\end{array}$ & $\begin{array}{r}1.13 \\
(1.00)\end{array}$ & $\begin{array}{r}8.52 \\
(9.00)\end{array}$ & $\begin{array}{r}19.88 \\
(20.25)\end{array}$ & 1.986 \\
\hline 2 & $45 / 10 / 45$ & $\begin{array}{r}19.51 \\
(20.25)\end{array}$ & $\begin{array}{r}38.09 \\
(40.50)\end{array}$ & $\begin{array}{l}11.15 \\
(9.00)\end{array}$ & $\begin{array}{r}19.51 \\
(20.25)\end{array}$ & $\begin{array}{l}10.53 \\
(9.00)\end{array}$ & $\begin{array}{r}1.21 \\
(1.00)\end{array}$ & 2.011 \\
\hline 3 & $10 / 45 / 45$ & $\begin{array}{r}1.33 \\
(1.00)\end{array}$ & $\begin{array}{r}9.69 \\
(9.00)\end{array}$ & $\begin{array}{l}10.03 \\
(9.00)\end{array}$ & $\begin{array}{r}19.39 \\
(20.25)\end{array}$ & $\begin{array}{r}39.46 \\
(40.50)\end{array}$ & $\begin{array}{r}20.06 \\
(20.25)\end{array}$ & 1.991 \\
\hline 4 & $40 / 40 / 20$ & $\begin{array}{r}15.18 \\
(16.00)\end{array}$ & $\begin{array}{r}15.82 \\
(16.00)\end{array}$ & $\begin{array}{r}32.91 \\
(32.00)\end{array}$ & $\begin{array}{r}3.79 \\
(4.00)\end{array}$ & $\begin{array}{r}15.82 \\
(16.00)\end{array}$ & $\begin{array}{r}16.45 \\
(16.00)\end{array}$ & 2.019 \\
\hline 5 & $40 / 20 / 40$ & $\begin{array}{r}16.12 \\
(16.00)\end{array}$ & $\begin{array}{r}31.72 \\
(32.00)\end{array}$ & $\begin{array}{r}15.59 \\
(16.00)\end{array}$ & $\begin{array}{r}15.85 \\
(16.00)\end{array}$ & $\begin{array}{r}16.12 \\
(16.00)\end{array}$ & $\begin{array}{r}4.56 \\
(4.00)\end{array}$ & 1.970 \\
\hline 6 & $20 / 40 / 40$ & $\begin{array}{r}4.01 \\
(4.00)\end{array}$ & $\begin{array}{r}15.66 \\
(16.00)\end{array}$ & $\begin{array}{r}16.46 \\
(16.00)\end{array}$ & $\begin{array}{r}16.05 \\
(16.00)\end{array}$ & $\begin{array}{r}31.72 \\
(32.00)\end{array}$ & $\begin{array}{r}16.05 \\
(16.00)\end{array}$ & 1.996 \\
\hline 7 & $35 / 35 / 30$ & $\begin{array}{r}12.82 \\
(12.25)\end{array}$ & $\begin{array}{r}20.94 \\
(21.00)\end{array}$ & $\begin{array}{r}25.91 \\
(24.50)\end{array}$ & $\begin{array}{r}6.80 \\
(9.00)\end{array}$ & $\begin{array}{r}20.41 \\
(21.00)\end{array}$ & $\begin{array}{r}13.08 \\
(12.25)\end{array}$ & 2.037 \\
\hline 8 & $35 / 30 / 35$ & $\begin{array}{r}12.01 \\
(12.25)\end{array}$ & $\begin{array}{r}23.72 \\
(24.50)\end{array}$ & $\begin{array}{r}22.22 \\
(21.00)\end{array}$ & $\begin{array}{r}12.31 \\
(12.25)\end{array}$ & $\begin{array}{r}22.22 \\
(21.00)\end{array}$ & $\begin{array}{r}7.50 \\
(9.00)\end{array}$ & 2.053 \\
\hline 9 & $30 / 35 / 35$ & $\begin{array}{r}6.96 \\
(9.00)\end{array}$ & $\begin{array}{r}20.88 \\
(21.00)\end{array}$ & $\begin{array}{r}21.51 \\
(21.00)\end{array}$ & $\begin{array}{r}12.66 \\
(12.25)\end{array}$ & $\begin{array}{r}25.32 \\
(24.50)\end{array}$ & $\begin{array}{r}12.66 \\
(12.25)\end{array}$ & 2.048 \\
\hline 10 & $20 / 20 / 60$ & $\begin{array}{r}3.97 \\
(4.00)\end{array}$ & $\begin{array}{r}23.35 \\
(24.00)\end{array}$ & $\begin{array}{r}8.33 \\
(8.00)\end{array}$ & $\begin{array}{r}35.34 \\
(36.00)\end{array}$ & $\begin{array}{r}24.62 \\
(24.00)\end{array}$ & $\begin{array}{r}4.36 \\
(4.00)\end{array}$ & 1.995 \\
\hline 11 & $20 / 60 / 20$ & $\begin{array}{r}4.03 \\
(4.00)\end{array}$ & $\begin{array}{r}8.87 \\
(8.00)\end{array}$ & $\begin{array}{r}23.38 \\
(24.00)\end{array}$ & $\begin{array}{r}4.03 \\
(4.00)\end{array}$ & $\begin{array}{r}23.79 \\
(24.00)\end{array}$ & $\begin{array}{r}35.88 \\
(36.00)\end{array}$ & 1.997 \\
\hline 12 & $60 / 20 / 20$ & $\begin{array}{r}35.86 \\
(36.00)\end{array}$ & $\begin{array}{r}23.36 \\
(24.00)\end{array}$ & $\begin{array}{r}23.64 \\
(24.00)\end{array}$ & $\begin{array}{r}4.07 \\
(4.00)\end{array}$ & $\begin{array}{r}8.69 \\
(8.00)\end{array}$ & $\begin{array}{r}4.34 \\
(4.00)\end{array}$ & 1.983 \\
\hline 13 & $33.3 / 33.3 / 33.3$ & $\begin{array}{r}11.32 \\
(11.11)\end{array}$ & $\begin{array}{r}21.90 \\
(22.22)\end{array}$ & $\begin{array}{r}22.25 \\
(22.22)\end{array}$ & $\begin{array}{r}10.95 \\
(11.11)\end{array}$ & $\begin{array}{r}22.62 \\
(22.22)\end{array}$ & $\begin{array}{r}10.95 \\
(11.11)\end{array}$ & 2.003 \\
\hline
\end{tabular}

$$
\begin{aligned}
R= & \ln \left(\frac{\boldsymbol{P}_{\mathrm{TT}} \cdot \boldsymbol{P}_{\mathrm{SS}}}{\boldsymbol{P}_{\mathrm{TS}} \cdot \boldsymbol{P}_{\mathrm{ST}}} \times \frac{\boldsymbol{P}_{\mathrm{TT}} \cdot \boldsymbol{P}_{\mathrm{FF}}}{\boldsymbol{P}_{\mathrm{TF}} \cdot \boldsymbol{P}_{\mathrm{FT}}} \times \frac{\boldsymbol{P}_{\mathrm{SS}} \cdot \boldsymbol{P}_{\mathrm{FF}}}{\boldsymbol{P}_{\mathrm{SF}} \cdot \boldsymbol{P}_{\mathrm{FS}}}\right) \\
= & \ln \frac{\left(1-\boldsymbol{P}_{\mathrm{TS}}-\boldsymbol{P}_{\mathrm{TF}}\right)\left(1-\boldsymbol{P}_{\mathrm{SF}}-\boldsymbol{P}_{\mathrm{ST}}\right)}{\boldsymbol{P}_{\mathrm{TS}} \cdot \boldsymbol{P}_{\mathrm{ST}}} \\
& \times \frac{\left(1-\boldsymbol{P}_{\mathrm{TS}}-\boldsymbol{P}_{\mathrm{TF}}\right)\left(1-\boldsymbol{P}_{\mathrm{FS}}-\boldsymbol{P}_{\mathrm{FT}}\right)}{\boldsymbol{P}_{\mathrm{TF}} \cdot \boldsymbol{P}_{\mathrm{FT}}}
\end{aligned}
$$

Table III. Sequence distributions in PET/S/F with various terpolymerization ratios

a Calculated values for random terpolymers are given in parenthesis. 
Sequence Distributions of Terpolyesters by NMR

Table IV. Sequence distributions in PET/S/M with various terpolymerization ratios ${ }^{\mathrm{a}}$

\begin{tabular}{|c|c|c|c|c|c|c|c|c|}
\hline Sample & $\begin{array}{c}\text { Feed ratios, } \\
\mathrm{T} / \mathbf{S} / \mathbf{M}\end{array}$ & $\mathrm{T}-\underset{\%}{\mathrm{E}-\mathrm{T}}$ & $\mathrm{T}-\underset{\%}{\mathrm{E}}-\mathrm{M}$, & $\underset{\%}{\mathbf{T}-\mathbf{E}-\mathbf{S},}$ & $\underset{\%}{\mathrm{M}-\mathrm{E}-\mathrm{M},}$ & $\underset{\%}{\mathbf{M}-\mathrm{E}-\mathrm{S},}$ & $\mathbf{S}-\mathbf{E}-\mathbf{S}$, & $B$ \\
\hline A & $40 / 40 / 20$ & $\begin{array}{r}15.87 \\
(16.00)\end{array}$ & $\begin{array}{r}15.81 \\
(16.00)\end{array}$ & $\begin{array}{r}32.33 \\
(32.00)\end{array}$ & $\begin{array}{r}3.90 \\
(4.00)\end{array}$ & $\begin{array}{r}15.87 \\
(16.00)\end{array}$ & $\begin{array}{r}16.19 \\
(16.00)\end{array}$ & 2.003 \\
\hline B & $35 / 35 / 30$ & $\begin{array}{r}11.00 \\
(12.25)\end{array}$ & $\begin{array}{r}22.13 \\
(21.00)\end{array}$ & $\begin{array}{r}26.13 \\
(24.50)\end{array}$ & $\begin{array}{r}7.68 \\
(9.00)\end{array}$ & $\begin{array}{r}21.90 \\
(21.00)\end{array}$ & $\begin{array}{r}11.14 \\
(12.25)\end{array}$ & 2.109 \\
\hline C & $30 / 30 / 40$ & $\begin{array}{r}8.75 \\
(9.00)\end{array}$ & $\begin{array}{r}23.60 \\
(24.00)\end{array}$ & $\begin{array}{r}17.76 \\
(18.00)\end{array}$ & $\begin{array}{r}15.81 \\
(16.00)\end{array}$ & $\begin{array}{r}24.81 \\
(24.00)\end{array}$ & $\begin{array}{r}9.24 \\
(9.00)\end{array}$ & 2.004 \\
\hline D & $45 / 45 / 10$ & $\begin{array}{r}20.39 \\
(20.25)\end{array}$ & $\begin{array}{r}9.48 \\
(9.00)\end{array}$ & $\begin{array}{r}39.84 \\
(40.50)\end{array}$ & $\begin{array}{r}0.95 \\
(1.00)\end{array}$ & $\begin{array}{r}9.01 \\
(9.00)\end{array}$ & $\begin{array}{r}20.30 \\
(20.25)\end{array}$ & 2.000 \\
\hline $\mathrm{E}$ & $30 / 20 / 50$ & $\begin{array}{r}9.12 \\
(9.00)\end{array}$ & $\begin{array}{r}28.61 \\
(30.00)\end{array}$ & $\begin{array}{r}12.57 \\
(12.00)\end{array}$ & $\begin{array}{r}23.89 \\
(25.00)\end{array}$ & $\begin{array}{r}21.38 \\
(20.00)\end{array}$ & $\begin{array}{r}4.41 \\
(4.00)\end{array}$ & 1.998 \\
\hline $\mathrm{F}$ & $50 / 30 / 20$ & $\begin{array}{r}24.64 \\
(25.00)\end{array}$ & $\begin{array}{r}19.43 \\
(20.00)\end{array}$ & $\begin{array}{r}30.33 \\
(30.00)\end{array}$ & $\begin{array}{r}4.26 \\
(4.00)\end{array}$ & $\begin{array}{r}12.32 \\
(12.00)\end{array}$ & $\begin{array}{r}9.00 \\
(9.00)\end{array}$ & 1.994 \\
\hline G & $50 / 20 / 30$ & $\begin{array}{r}25.35 \\
(25.00)\end{array}$ & $\begin{array}{r}29.09 \\
(30.00)\end{array}$ & $\begin{array}{r}19.78 \\
(20.00)\end{array}$ & $\begin{array}{r}8.72 \\
(9.00)\end{array}$ & $\begin{array}{r}12.88 \\
(12.00)\end{array}$ & $\begin{array}{r}4.15 \\
(4.00)\end{array}$ & 1.994 \\
\hline $\mathbf{H}$ & $20 / 50 / 30$ & $\begin{array}{r}4.33 \\
(4.00)\end{array}$ & $\begin{array}{r}11.93 \\
(12.00)\end{array}$ & $\begin{array}{r}20.50 \\
(20.00)\end{array}$ & $\begin{array}{r}8.18 \\
(9.00)\end{array}$ & $\begin{array}{r}30.02 \\
(30.00)\end{array}$ & $\begin{array}{r}25.02 \\
(25.00)\end{array}$ & 2.012 \\
\hline I & $30 / 50 / 20$ & $\begin{array}{r}9.67 \\
(9.00)\end{array}$ & $\begin{array}{r}12.44 \\
(12.00)\end{array}$ & $\begin{array}{r}29.49 \\
(30.00)\end{array}$ & $\begin{array}{r}3.68 \\
(4.00)\end{array}$ & $\begin{array}{r}20.27 \\
(20.00)\end{array}$ & $\begin{array}{r}24.42 \\
(25.00)\end{array}$ & 2.007 \\
\hline
\end{tabular}

a Calculated values for random terpolymers are given in parenthesis.

Table V. Linkage probabilities and degree of randomness in PET/S/F with various terpolymerization ratios

\begin{tabular}{|c|c|c|c|c|c|c|c|c|}
\hline Sample & $\begin{array}{c}\text { Feed ratios, } \\
\mathbf{T} / \mathbf{S} / \mathbf{F}\end{array}$ & $P_{\mathrm{ST}}$ & $\boldsymbol{P}_{\mathrm{TS}}$ & $\boldsymbol{P}_{\mathrm{TF}}$ & $P_{\mathrm{FT}}$ & $P_{\mathrm{SF}}$ & $\boldsymbol{P}_{\mathrm{FS}}$ & $R$ \\
\hline 1 & $45 / 45 / 10$ & 0.458 & 0.449 & 0.100 & 0.456 & 0.095 & 0.428 & $\ln 1.431$ \\
\hline 2 & $45 / 10 / 45$ & 0.462 & 0.126 & 0.432 & 0.434 & 0.437 & 0.120 & $\ln 0.701$ \\
\hline 3 & $10 / 45 / 45$ & 0.112 & 0.448 & 0.432 & 0.111 & 0.440 & 0.448 & $\ln 1.252$ \\
\hline 4 & $40 / 40 / 20$ & 0.403 & 0.416 & 0.200 & 0.403 & 0.194 & 0.403 & $\ln 0.854$ \\
\hline 5 & $40 / 20 / 40$ & 0.381 & 0.196 & 0.398 & 0.399 & 0.394 & 0.202 & $\ln 1.564$ \\
\hline 6 & $20 / 40 / 40$ & 0.205 & 0.410 & 0.390 & 0.197 & 0.395 & 0.399 & $\ln 1.025$ \\
\hline 7 & $35 / 35 / 30$ & 0.357 & 0.357 & 0.289 & 0.381 & 0.282 & 0.371 & $\ln 0.684$ \\
\hline 8 & $35 / 30 / 35$ & 0.374 & 0.318 & 0.338 & 0.335 & 0.374 & 0.314 & $\ln 0.591$ \\
\hline 9 & $30 / 35 / 35$ & 0.298 & 0.382 & 0.371 & 0.292 & 0.351 & 0.354 & $\ln 0.614$ \\
\hline 10 & $20 / 20 / 60$ & 0.200 & 0.210 & 0.588 & 0.197 & 0.592 & 0.208 & $\ln 1.103$ \\
\hline 11 & $20 / 60 / 20$ & 0.197 & 0.580 & 0.220 & 0.217 & 0.200 & 0.583 & $\ln 0.917$ \\
\hline 12 & $60 / 20 / 20$ & 0.577 & 0.200 & 0.197 & 0.581 & 0.212 & 0.216 & $\ln 1.103$ \\
\hline 13 & $33.3 / 33.3 / 33.3$ & 0.333 & 0.333 & 0.327 & 0.330 & 0.339 & 0.341 & $\ln 1.009$ \\
\hline
\end{tabular}

Table VI. Linkage probabilities and degree of randomness in PET/S/M with various terpolymerization ratios

\begin{tabular}{|c|c|c|c|c|c|c|c|c|}
\hline Sample & $\begin{array}{l}\text { Feed ratios, } \\
\mathrm{T} / \mathrm{S} / \mathrm{M}\end{array}$ & $\boldsymbol{P}_{\mathrm{ST}}$ & $\boldsymbol{P}_{\mathrm{TS}}$ & $\boldsymbol{P}_{\mathrm{TM}}$ & $\boldsymbol{P}_{\mathrm{MT}}$ & $S_{\mathrm{SM}}$ & $\boldsymbol{P}_{\mathrm{MS}}$ & $R$ \\
\hline A & $40 / 40 / 20$ & 0.401 & 0.404 & 0.198 & 0.401 & 0.197 & 0.402 & $\ln 0.975$ \\
\hline B & $35 / 35 / 30$ & 0.371 & 0.372 & 0.315 & 0.372 & 0.311 & 0.368 & $\ln 0.366$ \\
\hline $\mathrm{C}$ & $30 / 30 / 40$ & 0.291 & 0.301 & 0.401 & 0.294 & 0.407 & 0.310 & $\ln 0.981$ \\
\hline D & $45 / 45 / 10$ & 0.445 & 0.442 & 0.105 & 0.465 & 0.101 & 0.442 & $\ln 0.836$ \\
\hline E & $30 / 20 / 50$ & 0.294 & 0.211 & 0.482 & 0.293 & 0.499 & 0.219 & $\ln 0.992$ \\
\hline F & $50 / 30 / 20$ & 0.500 & 0.306 & 0.196 & 0.483 & 0.203 & 0.306 & $\ln 1.085$ \\
\hline G & $50 / 20 / 30$ & 0.483 & 0.199 & 0.292 & 0.489 & 0.314 & 0.217 & $\ln 0.992$ \\
\hline $\mathbf{H}$ & $20 / 50 / 30$ & 0.205 & 0.498 & 0.290 & 0.204 & 0.300 & 0.515 & $\ln 0.940$ \\
\hline I & $30 / 50 / 20$ & 0.299 & 0.482 & 0.203 & 0.311 & 0.206 & 0.506 & $\ln 0.872$ \\
\hline
\end{tabular}




\section{Murano}

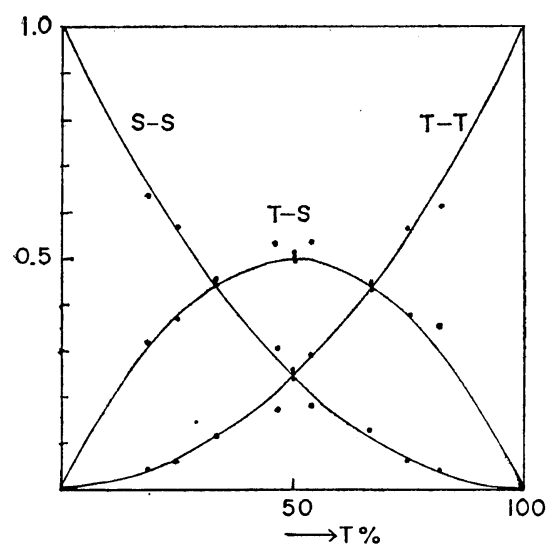

(a)

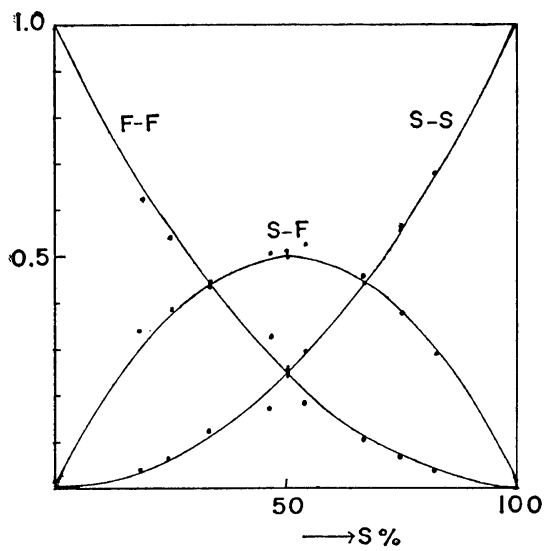

(b)

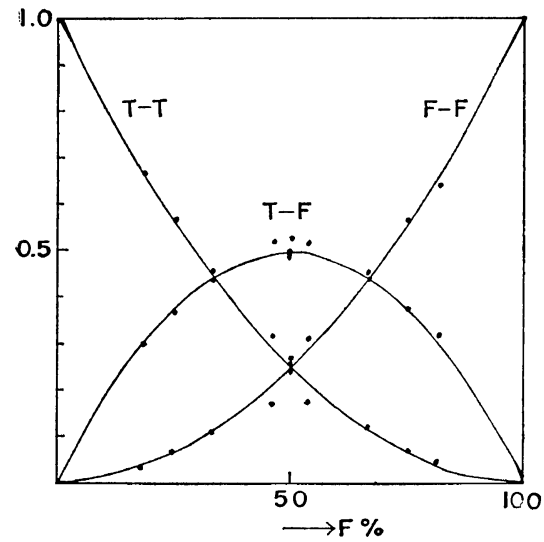

(c)

Figure 5. Relationships between ((a) $P_{\mathrm{T}}$ and $\boldsymbol{P}_{\mathrm{T}-\mathrm{E}-\mathrm{T}}, \boldsymbol{P}_{\mathrm{T}-\mathrm{E}-\mathrm{S}}, \boldsymbol{P}_{\mathrm{S}-\mathrm{E}-\mathrm{S}} ;$ (b) $\boldsymbol{P}_{\mathrm{S}}$ and $\boldsymbol{P}_{\mathrm{S}-\mathrm{E}-\mathrm{S}}$, $\boldsymbol{P}_{\mathrm{S}-\mathrm{E}-\mathrm{F}}, \boldsymbol{P}_{\mathrm{F}-\mathrm{E}-\mathrm{F}} ;$ (c) $\boldsymbol{P}_{\mathrm{F}}$ and $\boldsymbol{P}_{\mathrm{F}-\mathrm{E}-\mathrm{F}}, \boldsymbol{P}_{\mathrm{F}-\mathrm{E}-\mathrm{T}}$, $\left.\boldsymbol{P}_{\mathrm{T}-\mathrm{E}-\mathrm{T}}\right)$ in $\mathrm{PET} / \mathrm{S} / \mathrm{F}$ terpolymers.

$$
\times \frac{\left(1-\boldsymbol{P}_{\mathrm{ST}}-\boldsymbol{P}_{\mathrm{SF}}\right)\left(1-\boldsymbol{P}_{\mathrm{FS}}-\boldsymbol{P}_{\mathrm{FT}}\right)}{\boldsymbol{P}_{\mathrm{SF}} \cdot \boldsymbol{P}_{\mathrm{FS}}}
$$

In the case of a random (or Bernoullian) terpolymer, where $\boldsymbol{P}_{\mathrm{TS}}=\boldsymbol{P}_{\mathrm{FS}}=\boldsymbol{P}_{\mathrm{S}}, \quad \boldsymbol{P}_{\mathrm{TF}}=\boldsymbol{P}_{\mathrm{SF}}=\boldsymbol{P}_{\mathrm{F}}$, and $\boldsymbol{P}_{\mathrm{FT}}=\boldsymbol{P}_{\mathrm{ST}}=\boldsymbol{P}_{\mathrm{T}}, \boldsymbol{R}$ takes 0 . These values are obtained for $\mathrm{PET} / \mathrm{S} / \mathrm{F}$ terpolymers as shown in Table V, and for PET/S/M terpolymers in Table VI. These tables also show that these terpolymers are in random (or Bernoullian) distribution.

This can be seen more clearly in Figures 5 and 6 . In Figure 5 a, distributions of terephthalate and sebacate are shown by normalizing

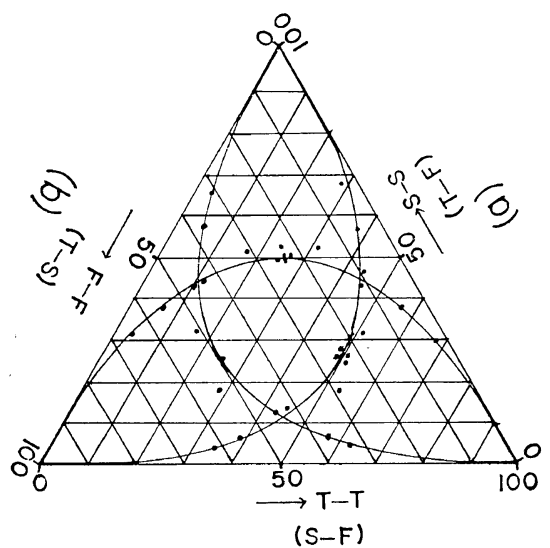

(c)

Figure 6. Relationships between ((a) $P_{\mathrm{T}-\mathrm{E}-\mathrm{T}}$, $\boldsymbol{P}_{\mathrm{T}-\mathrm{E}-\mathrm{S}}$, and $\boldsymbol{P}_{\mathrm{S}-\mathrm{E}-\mathrm{S}}$; (b) $\boldsymbol{P}_{\mathrm{S}-\mathrm{E}-\mathrm{S}}, \boldsymbol{P}_{\mathrm{S}-\mathrm{E}-\mathrm{F}}$, and $\boldsymbol{P}_{\mathrm{F}-\mathrm{E}-\mathrm{F}}$; (c) $\boldsymbol{P}_{\mathrm{F}-\mathrm{E}-\mathrm{F}}, \boldsymbol{P}_{\mathrm{F}-\mathrm{E}-\mathrm{T}}$, and $\left.\boldsymbol{P}_{\mathrm{T}-\mathrm{E}-\mathrm{T}}\right)$ in $\mathrm{PET} / \mathrm{S} / \mathrm{F}$ terpolymers in a triangular coordinate system.

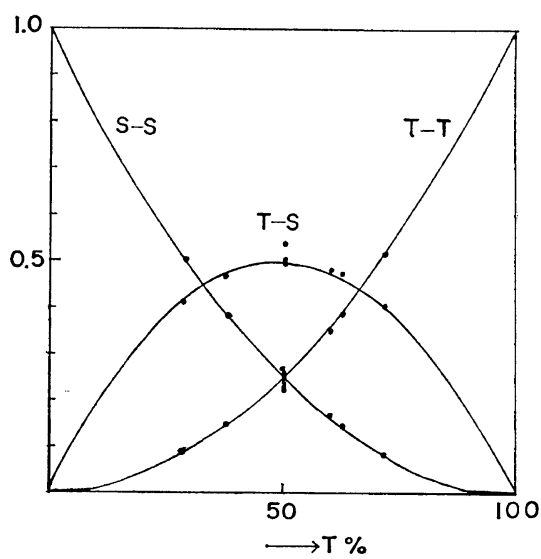

(a)

Polymer, J., Vol. 1, No. 6, 1970 


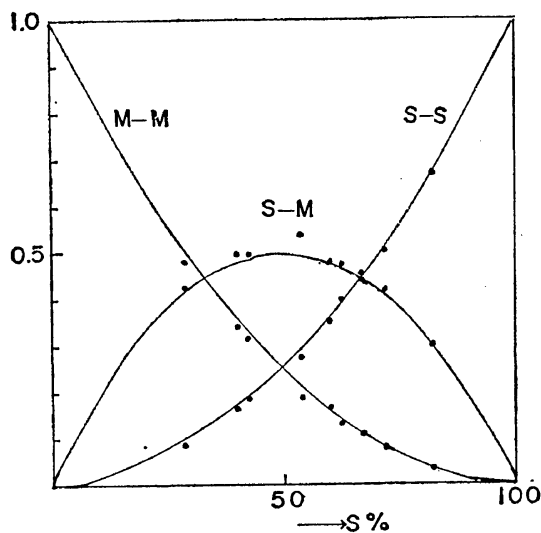

(b)

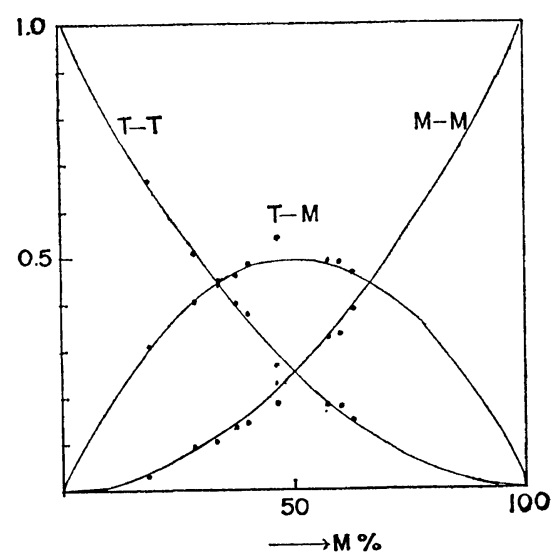

(c)

Figure 7. Relationships between $\left((\mathrm{a}) \boldsymbol{P}_{\mathrm{T}} \quad\right.$ and $\boldsymbol{P}_{\mathrm{T}-\mathrm{E}-\mathrm{T}}, \boldsymbol{P}_{\mathrm{T}-\mathrm{E}-\mathrm{S}}, \boldsymbol{P}_{\mathrm{S}-\mathrm{E}-\mathrm{S}} ;$ (b) $\boldsymbol{P}_{\mathrm{S}}$ and $\boldsymbol{P}_{\mathrm{S}-\mathrm{E}-\mathrm{S}}$, $\boldsymbol{P}_{\mathrm{S}-\mathrm{E}-\mathrm{M}}, \boldsymbol{P}_{\mathrm{M}-\mathrm{E}-\mathrm{M}}$; (c) $\boldsymbol{P}_{\mathrm{M}}$ and $\boldsymbol{P}_{\mathrm{M}-\mathrm{E}-\mathrm{M}}, \boldsymbol{P}_{\mathrm{M}-\mathrm{E}-\mathrm{T}}$, $\boldsymbol{P}_{\mathbf{T}-\mathrm{E}-\mathrm{T})}$ in $\mathrm{PET} / \mathrm{S} / \mathbf{M}$ terpolymers.

$\boldsymbol{P}_{\mathrm{T}-\mathrm{E}-\mathrm{T}}, \boldsymbol{P}_{\mathrm{T}-\mathrm{E}-\mathrm{S}}$, and $\boldsymbol{P}_{\mathrm{S}-\mathrm{E}-\mathrm{S}} . \quad \mathrm{T}$ and $\mathrm{S}$ are found in random (or Bernoullian) distribution. Similar relationships of sebacate and fumarate are shown in Figure $5 \mathrm{~b}$ by normalizing $\boldsymbol{P}_{\mathrm{S}-\mathrm{E}-\mathrm{S}}, \boldsymbol{P}_{\mathrm{S}-\mathrm{E}-\mathrm{F}}$, and $\boldsymbol{P}_{\mathrm{F}-\mathrm{E}-\mathrm{F}}$, and those of fumarate and terephthalate in Figure $5 \mathrm{c}$, where $\boldsymbol{P}_{\mathrm{F}-\mathrm{E}-\mathrm{F}}, \boldsymbol{P}_{\mathrm{F}-\mathrm{E}-\mathrm{T}}$, and $\boldsymbol{P}_{\mathrm{T}-\mathrm{E}-\mathrm{T}}$ are normalized. Solid curves in these Figures mean random (or Bernoullian) distributions of these acid groups. These relationships are also shown in Figure 6 with the triangular coordinate system, where relationships between $\boldsymbol{P}_{\mathrm{T}-\mathrm{E}-\mathrm{T}}, \boldsymbol{P}_{\mathrm{T}-\mathrm{E}-\mathrm{S}}$, and $\boldsymbol{P}_{\mathrm{S}-\mathrm{E}-\mathrm{S}}(\mathrm{a})$, and those of $\boldsymbol{P}_{\mathrm{S}-\mathrm{E}-\mathrm{S}}, \boldsymbol{P}_{\mathrm{S}-\mathrm{E}-\mathrm{F}}$, and $\boldsymbol{P}_{\mathrm{F}-\mathrm{E}-\mathrm{F}}(\mathrm{b})$, and

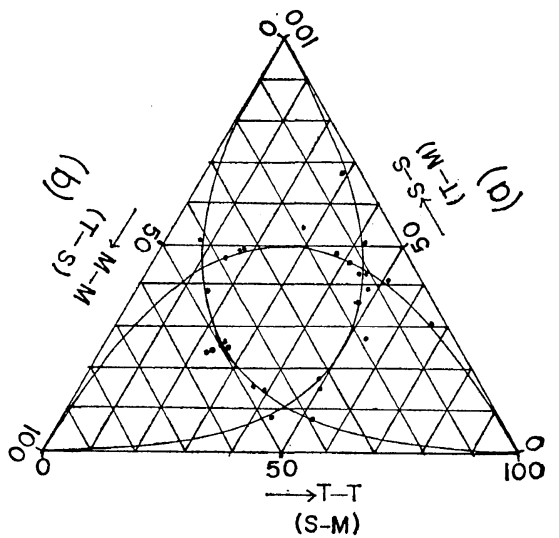

(c)

Figure 8. Relationships between ((a) $\boldsymbol{P}_{\mathrm{T}-\mathrm{E}-\mathrm{T}}$, $\boldsymbol{P}_{\mathrm{T}-\mathrm{E}-\mathrm{S}}$, and $\boldsymbol{P}_{\mathrm{S}-\mathrm{E}-\mathrm{S}}$; (b) $\boldsymbol{P}_{\mathrm{S}-\mathrm{E}-\mathrm{S}}, \boldsymbol{P}_{\mathrm{S}-\mathrm{E}-\mathrm{M}}$, and $\boldsymbol{P}_{\mathrm{M}-\mathrm{E}-\mathrm{M}}$; (c) $\boldsymbol{P}_{\mathrm{M}-\mathrm{E}-\mathrm{M}}, \boldsymbol{P}_{\mathrm{M}-\mathrm{E}-\mathrm{T}}$, and $\left.\boldsymbol{P}_{\mathrm{T}-\mathrm{E}-\mathrm{T}}\right)$ in $\mathbf{P E T} / \mathrm{S} / \mathrm{M}$ terpolymers in a triangular coordinate system.

those of $\boldsymbol{P}_{\mathrm{F}-\mathrm{E}-\mathrm{F}}, \boldsymbol{P}_{\mathrm{F}-\mathrm{E}-\mathrm{T}}$, and $\boldsymbol{P}_{\mathrm{T}-\mathrm{E}-\mathrm{T}}(\mathrm{c})$, are shown, the solid curves meaning random (or Bernoullian) distributions of terephthalate and sebacate, sebacate and fumarate, and fumarate and terephthalate, respectively. From these figures, it is quite clear that terephthalate, sebacate, and fumarate are in random (or Bernoullian) distributions in these terpolyesters.

Similar relationships of PET/S/M are plotted in Figures 7 and 8 by normalizing $\boldsymbol{P}_{\mathrm{T}-\mathrm{E}-\mathrm{T}}$, $\boldsymbol{P}_{\mathrm{T}-\mathrm{E}-\mathrm{S}}$, and $\boldsymbol{P}_{\mathrm{S}-\mathrm{E}-\mathrm{S}}$ (Figure 7 a), $\boldsymbol{P}_{\mathrm{S}-\mathrm{E}-\mathrm{S}}, \boldsymbol{P}_{\mathrm{S}-\mathrm{E}-\mathrm{M}}$, and $\boldsymbol{P}_{\mathrm{M}-\mathrm{E}-\mathrm{M}}$ (Figure $7 \mathrm{~b}$ ), and $\boldsymbol{P}_{\mathrm{M}-\mathrm{E}-\mathrm{M}}, \boldsymbol{P}_{\mathrm{M}-\mathrm{E}-\mathrm{T}}$, and $P_{\mathrm{T}-\mathrm{E}-\mathrm{T}}$ (Figure $7 \mathrm{c}$ ). These figures clearly show that terephthalate, sebacate, and malonate are randomly distributed in these terpolyesters.

It is quite useful to plot in the triangular coordinate system in studying the sequence distribution of terpolymers.

Acknowledgement. The author wishes to express his sincere thanks to Dr. S. Satoh of Nippon Electric Varian, Ltd., for measurement of the $100-\mathrm{MHz}$ NMR spectra and advice, and to Dr. R. Yamadera of the same company for helpful discussions.

\section{REFERENCES}

1. R. C. Ferguson, J. Amer. Chem. Soc., 82, 2416 (1960). 


\section{Murano}

2. R. Yamadera and M. Murano, J. Polym. Sci., Part A-1, 5, 2259 (1967).

3. T. Harada and N. Ueda, Kobunshi Kagaku (Chem. High Polymers), 22, 685 (1965).

4. T. S. Khramova, Ya. G. Urman, O. A. Mochalova, F. M. Medvedeva, and I. Ya. Slonim, Vysokomol. Soed., Ser. A, 10, 894 (1968).

5. T. Kagiya, M. Izu, T. Matsuda, M. Matsuda, and K. Fukui, J. Polym. Sci., Part A-1, 6, 2059
(1968).

6. M. Murano and R. Yamadera, Polymer J., to be published.

7. M. Murano, J. Polym. Sci., Part B, to be published.

8. R. Chûjô, J. Phys. Soc. Japan, 21, 2669 (1966).

9. H. J. Harwood and W. M. Ritchey, J. Polym. Sci., Part B, 2, 601 (1964).

10. M. H. Thiel, Macromolecules, 2, 137 (1969). 